\title{
Data at the moment of infinite expansion for polarized Gowdy
}

\author{
H Ringström ${ }^{1}$ \\ Max-Planck-Institut för Gravitationsphysik, Am Mühlenberg 1, D-14476 Golm, Germany \\ E-mail: hansr@math.kth.se
}

Received 24 November 2004, in final form 15 March 2005

Published 6 April 2005

Online at stacks.iop.org/CQG/22/1647

\begin{abstract}
In a recent paper by Thomas Jurke, it was proved that the asymptotic behaviour of a solution to the polarized Gowdy equation in the expanding direction is of the form $\alpha \ln t+\beta+t^{-1 / 2} v+O\left(t^{-3 / 2}\right)$, where $\alpha$ and $\beta$ are constants and $v$ is a solution to the standard wave equation with zero mean value. Furthermore, it was proved that $\alpha, \beta$ and $v$ uniquely determine the solution. Here we wish to point out that given $\alpha, \beta$ and $v$ with the above properties, one can construct a solution to the polarized Gowdy equation with the above asymptotics. In other words, we show that $\alpha, \beta$ and $v$ constitute data at the moment of infinite expansion. We then use this fact to make the observation that there are polarized Gowdy spacetimes such that in the areal time coordinate, the quotient of the maximum and the minimum of the mean curvature on a constant time hypersurface is unbounded as time tends to infinity.
\end{abstract}

PACS number: 04.20.Ex

\section{Introduction}

\subsection{Motivation}

Recently, conjectures have appeared concerning the asymptotic behaviour of solutions to Einstein's equations in the expanding direction of cosmological spacetimes (cf $[1,3]$, and references therein). The conjectures are usually phrased in terms of a constant mean curvature foliation. In some situations, it is however of interest to consider other foliations, and this motivates the question of what the relation is between the asymptotic behaviour in the different foliations. To try to obtain results in all generality is unrealistic. A problem which is more reasonable is to consider subclasses of spacetimes with symmetries. One subclass which has attracted attention is the Gowdy spacetimes. In this case, there is a two-dimensional group

1 Present address: Department of Mathematics, KTH, 10044 Stockholm, Sweden. 
of isometries that acts on spatial hypersurfaces, so that the relevant equations are a system of hyperbolic equations in $(1+1)$ dimensions. Most of the work concerning this class has been done in the so-called areal time coordinate. The corresponding foliation does not have constant mean curvature hypersurfaces, but it has a natural geometric definition. It is thus of interest to get a feeling for what the relation is between the constant mean curvature foliation and the areal time coordinate foliation in Gowdy spacetimes. One conjecture one could formulate is that all reasonable foliations should behave in a similar way in the asymptotic regimes. It is then natural to ask if the quotient of the maximum and the minimum of the absolute value of the mean curvature of the hypersurfaces of constant areal time is bounded in the expanding direction. If it is not bounded, we have an indication that there is a difference between the two foliations.

A curious fact concerning the Gowdy spacetimes is that it is in some sense possible to prescribe data 'at the singularity' (cf $[5,6]$ ). The data give the leading-order asymptotic behaviour close to the singularity and uniquely determine the solution. It is of some interest to find out if it is possible to do something similar in the expanding direction.

\subsection{Gowdy spacetimes}

The Gowdy spacetimes are a class of vacuum spacetimes with a two-dimensional group of isometries. Of the spatial topologies compatible with the symmetry requirements, only $T^{3}$ is expected to be compatible with infinite expansion. For this reason we shall only be interested in such a spatial topology in this paper. There are natural conditions defining the Gowdy spacetimes (see [7] and references therein), but we shall not write them down here. For the purposes of the present paper, a $T^{3}$-Gowdy spacetime is defined as a Lorentz manifold $\mathbb{R}_{+} \times T^{3}$, where $\mathbb{R}_{+}=(0, \infty)$, with metric

$g=t^{-1 / 2} \mathrm{e}^{\lambda / 2}\left(-\mathrm{d} t^{2}+\mathrm{d} \theta^{2}\right)+t\left[\mathrm{e}^{P} \mathrm{~d} \sigma^{2}+2 \mathrm{e}^{P} Q \mathrm{~d} \sigma \mathrm{d} \delta+\left(\mathrm{e}^{\mathrm{P}} \mathrm{Q}^{2}+\mathrm{e}^{-\mathrm{P}}\right) \mathrm{d} \delta^{2}\right]$,

where $P, Q$ and $\lambda$ only depend on $t$ and $\theta$, satisfying Einstein's vacuum equations. In terms of $P, Q$ and $\lambda$, the equations are

$$
\begin{aligned}
& P_{t t}+\frac{1}{t} P_{t}-P_{\theta \theta}-\mathrm{e}^{2 P}\left(Q_{t}^{2}-Q_{\theta}^{2}\right)=0 \\
& Q_{t t}+\frac{1}{t} Q_{t}-Q_{\theta \theta}+2\left(P_{t} Q_{t}-P_{\theta} Q_{\theta}\right)=0,
\end{aligned}
$$

and

$$
\begin{aligned}
& \lambda_{t}=t\left[P_{t}^{2}+P_{\theta}^{2}+\mathrm{e}^{2 P}\left(Q_{t}^{2}+Q_{\theta}^{2}\right)\right] \\
& \lambda_{\theta}=2 t\left(P_{\theta} P_{t}+\mathrm{e}^{2 P} Q_{\theta} Q_{t}\right) .
\end{aligned}
$$

Note that translations in $\sigma$ and $\delta$ constitute isometries of the solution, and in this way we obtain an action by isometries of $T^{2}$ on the spacetime. The area of the orbit obtained by letting $T^{2}$ act in this way is $t$, hence the name areal time coordinate. It is important to note that equations (2) and (3) do not depend upon $\lambda$. In practice, one can thus analyse the behaviour of solutions to these equations separately, and then deduce consequences for $\lambda$. Note that the initial data for $P$ and $Q$ have to satisfy a condition for this procedure to make sense, namely, the integral of the right-hand side of (5) has to vanish. This is however the only restriction on the choice of initial data. Here we are interested in the polarized subcase which is defined by $Q=0$. The two nonlinear equations (2) and (3) then simply become one linear equation. We shall refer to (2) with the terms involving $Q$ set equal to zero as the polarized Gowdy equation. 


\subsection{Results}

Due to results of [4], each solution to the polarized Gowdy equation can be written as

$$
P=\alpha \ln t+\beta+t^{-1 / 2} v+\psi
$$

where $v$ is a solution of the flat wave equation, $\psi=O\left(t^{-3 / 2}\right)$ and similarly for the first derivatives of $\psi$. Note that this result was already announced in [2], though no proof was given. The result above tells us that given a solution to the polarized equation, there is a solution to the flat wave equation $v$ such that (6) holds. It is then of interest to find out if one is actually allowed to specify $v$. This turns out to be the case.

Proposition 1. Let $\alpha, \beta \in \mathbb{R}$ and let $v \in C^{\infty}\left(\mathbb{R}_{+} \times S^{1}, \mathbb{R}\right)$ satisfy

$$
v_{t t}-v_{\theta \theta}=0 \quad \text { and } \quad \int_{S^{1}} v(t, \theta) \mathrm{d} \theta=0,
$$

the last equation being valid for all $t \in \mathbb{R}_{+}$. Then there exists a unique

$$
\psi \in C^{\infty}\left(\mathbb{R}_{+} \times S^{1}, \mathbb{R}\right), \quad \int_{S^{1}} \psi(t, \theta) \mathrm{d} \theta=0,
$$

the last equation being valid for all $t \in \mathbb{R}_{+}$, such that first derivatives of $\psi$ are $o\left(t^{-1 / 2}\right)$ in $L^{2}$ as time tends to infinity and such that $P$ defined by (6) satisfies

$$
P_{t t}+\frac{1}{t} P_{t}-P_{\theta \theta}=0
$$

The constructed $\psi$ has the property that it and all its derivatives are of the order $O\left(t^{-3 / 2}\right)$ as time tends to infinity. Finally, if

$$
\int_{S^{1}}\left(v_{t} v_{\theta}\right)(t, \theta) \mathrm{d} \theta=0
$$

(note that the left-hand side is independent of time due to (7)), the integral of the right-hand side of (5) is zero, so that $\lambda$ is well defined, and (1), where $Q$ is set equal to zero, defines a solution to Einstein's vacuum equations.

Remark. It would of course be interesting to prove a similar result in general. Even though there is information concerning the mean value in this case (cf [7]), it is however not so clear what the exact statement should be.

An immediate corollary to this statement is the following.

Corollary 1. There is a solution $P$ to (2) and (3) with $Q=0$ and a solution $\lambda$ to (4) and (5) with $Q=0$ such that the following holds: there is a sequence $t_{l} \rightarrow \infty$ and sequences $\theta_{1, l}, \theta_{2, l} \in S^{1}$ such that if $k$ is the second fundamental form of constant $t$ hypersurfaces, then

$$
\lim _{l \rightarrow \infty} \frac{-(\operatorname{tr} k)\left(t_{l}, \theta_{2, l}\right)}{-(\operatorname{tr} k)\left(t_{l}, \theta_{1, l}\right)}=\infty .
$$

Remark. Regardless of convention, tr $k$ is always bounded away from zero on a fixed constant $t$ hypersurface. 


\section{Proofs}

Let us start by proving that one can specify data at the moment of infinite expansion.

Proof of proposition 1. That $P$ satisfies (9), where $P$ is given by (6) and $v$ satisfies (7), is equivalent to $\psi$ satisfying

$$
\psi_{t t}+\frac{1}{t} \psi_{t}-\psi_{\theta \theta}=-\frac{1}{4} t^{-5 / 2} \nu
$$

Given initial data for some $T>0$, one can of course solve this equation. The problem is to ensure that the solution has the desired asymptotics. What we wish to do is to put zero initial data at infinity. We do this by considering a sequence of solutions each defined by setting the initial data to zero at a later and later time.

Consider a solution to (11) defined by giving zero initial data at $t=T \geqslant 1$. In other words, we demand that $\psi=\psi_{t}=0$ at $t=T$. Due to the second equation of (7) and (11), we obtain

$$
\partial_{t}\left[t \int_{S^{1}} \psi_{t} \mathrm{~d} \theta\right]=0
$$

This implies that

$$
t \int_{S^{1}} \psi_{t} \mathrm{~d} \theta=c_{3}
$$

for some constant $c_{3}$. Since the initial data at $t=T$ are zero, this constant has to be zero. Dividing the equality by $t$, we obtain

$$
\int_{S^{1}} \psi_{t} \mathrm{~d} \theta=0
$$

Integrating this inequality in time, we obtain the conclusion that

$$
\int_{S^{1}} \psi \mathrm{d} \theta=c_{4}
$$

for some constant $c_{4}$. Again, due to the fact that the initial data are zero at $t=T$, we obtain $c_{4}=0$. We get the conclusion that the second property in (8) holds for all $t$. Let us define

$$
\begin{aligned}
& H=\frac{1}{2} \int_{S^{1}}\left(\psi_{t}^{2}+\psi_{\theta}^{2}\right) \mathrm{d} \theta \\
& \Gamma=\frac{1}{2 t} \int_{S^{1}} \psi \psi_{t} \mathrm{~d} \theta .
\end{aligned}
$$

Note that for $t \geqslant 1$,

$$
|\Gamma| \leqslant \frac{1}{2 t} H, \quad \frac{1}{2} H \leqslant H+\Gamma \leqslant \frac{3}{2} H .
$$

The second inequality is a consequence of the first and the fact that we are only interested in the region $t \geqslant 1$. In order to prove the first inequality, note that

$$
|\Gamma|=\frac{1}{2 t}\left|\int_{S^{1}} \psi \psi_{t} \mathrm{~d} \theta\right| \leqslant \frac{1}{4 t} \int_{S^{1}}\left[\psi^{2}+\psi_{t}^{2}\right] \mathrm{d} \theta,
$$

due to the inequality $a b \leqslant\left(a^{2}+b^{2}\right) / 2$ which holds for all real numbers $a, b$. Furthermore, let $\chi$ be any smooth $2 \pi$ periodic function. Due to periodicity, there is an expansion

$$
\chi(\theta)=\sum_{n \in \mathbb{Z}} a_{n} \mathrm{e}^{\mathrm{in} \theta} .
$$


If the mean value of $\chi$ is zero, then $a_{0}=0$ and we obtain the consequence that

$$
\int_{S^{1}}|\chi|^{2}(\theta) \mathrm{d} \theta=2 \pi \sum_{n \in \mathbb{Z}}\left|a_{n}\right|^{2} \leqslant 2 \pi \sum_{n \in \mathbb{Z}} n^{2}\left|a_{n}\right|^{2}=\int_{S^{1}}\left|\chi^{\prime}\right|^{2}(\theta) \mathrm{d} \theta .
$$

In particular,

$$
\int_{S^{1}} \psi^{2} \mathrm{~d} \theta \leqslant \int_{S^{1}} \psi_{\theta}^{2} \mathrm{~d} \theta
$$

Combining this observation with (15), we obtain

$$
|\Gamma| \leqslant \frac{1}{4 t} \int_{S^{1}}\left[\psi_{\theta}^{2}+\psi_{t}^{2}\right] \mathrm{d} \theta=\frac{1}{2 t} H,
$$

which proves the first inequality in (14).

Compute

$$
\begin{aligned}
\frac{\mathrm{d} H}{\mathrm{~d} t} & =-\frac{1}{t} \int_{S^{1}} \psi_{t}^{2} \mathrm{~d} \theta-\frac{1}{4} t^{-5 / 2} \int_{S^{1}} \nu \psi_{t} \mathrm{~d} \theta \\
\frac{\mathrm{d} \Gamma}{\mathrm{d} t} & =-\frac{2}{t} \Gamma+\frac{1}{2 t} \int_{S^{1}}\left(\psi_{t}^{2}-\psi_{\theta}^{2}\right) \mathrm{d} \theta-\frac{1}{8} t^{-7 / 2} \int_{S^{1}} \nu \psi \mathrm{d} \theta .
\end{aligned}
$$

Due to (14) and the fact that $t \geqslant 1$, we have

$$
\frac{1}{t}|\Gamma| \leqslant \frac{1}{t^{2}}(H+\Gamma) \text {. }
$$

Furthermore,

$\int_{S^{1}}\left|\nu \psi_{t}\right| \mathrm{d} \theta \leqslant \sqrt{2}\|\nu\|_{L^{2}\left(S^{1}, \mathbb{R}\right)} H^{1 / 2}, \quad \int_{S^{1}}|\nu \psi| \mathrm{d} \theta \leqslant \sqrt{2}\|v\|_{L^{2}\left(S^{1}, \mathbb{R}\right)} H^{1 / 2}$,

where the last inequality is due to the fact that the mean value of $\psi$ is zero. Combining the above equations and inequalities, we get the conclusion that

$$
\frac{\mathrm{d}(H+\Gamma)}{\mathrm{d} t} \geqslant-\left(\frac{1}{t}+\frac{1}{t^{2}}\right)(H+\Gamma)-t^{-5 / 2}\|\nu\|_{L^{2}\left(S^{1}, \mathbb{R}\right)}(H+\Gamma)^{1 / 2}
$$

where we have used the fact that $t \geqslant 1$. Define

$$
\mathcal{E}=t \mathrm{e}^{-1 / t}(H+\Gamma) \text {. }
$$

We have

$$
\frac{\mathrm{d} \mathcal{E}}{\mathrm{d} t} \geqslant-t^{1 / 2} \mathrm{e}^{-1 /(2 t)} t^{-5 / 2}\|v\|_{L^{2}\left(S^{1}, \mathbb{R}\right)} \mathcal{E}^{1 / 2} \geqslant-t^{-2}\|v\|_{L^{2}\left(S^{1}, \mathbb{R}\right)} \mathcal{E}^{1 / 2}
$$

Let $T_{1} \in[1, T]$ and integrate the above inequality from $T_{1}$ to $T$, keeping in mind that $\mathcal{E}(T)=0$. We get

$$
\mathcal{E}^{1 / 2}\left(T_{1}\right) \leqslant \frac{1}{2} \int_{T_{1}}^{T} t^{-2}\|v\|_{L^{2}\left(S^{1}, \mathbb{R}\right)} \mathrm{d} t
$$

Let

$$
\|v\|_{L^{\infty} L^{2}\left(\mathbb{R}_{+} \times S^{1}, \mathbb{R}\right)}=\sup _{t \in \mathbb{R}_{+}}\|v(t, \cdot)\|_{L^{2}\left(S^{1}, \mathbb{R}\right)} .
$$

Note that this is finite due to the fact that

$$
\int_{S^{1}}\left(v_{t}^{2}+v_{\theta}^{2}\right) \mathrm{d} \theta
$$


is constant in time and since the $L^{2}$-norm of $v$ is dominated by the $L^{2}$-norm of $v_{\theta}$ due to the fact that the mean value of $v$ is zero. We get

$$
\mathcal{E}\left(T_{1}\right) \leqslant \frac{1}{4}\|\nu\|_{L^{\infty} L^{2}\left(\mathbb{R}_{+} \times S^{1}, \mathbb{R}\right)}^{2} T_{1}^{-2},
$$

so that

$$
H\left(T_{1}\right) \leqslant \frac{\mathrm{e}}{2}\|\nu\|_{L^{\infty} L^{2}\left(\mathbb{R}_{+} \times S^{1}, \mathbb{R}\right)}^{2} T_{1}^{-3} .
$$

Note in particular that the bound is independent of $T$. Furthermore, the construction behaves well under differentiation of $\psi$ with respect to $\theta$. In fact, $\partial_{\theta}^{k} \psi$ has the same properties as $\psi$, we need only replace $v$ with $\partial_{\theta}^{k} v$, which is of course a solution to the wave equation. In particular, if we define

$$
H_{k}=\frac{1}{2} \int_{S^{1}}\left[\left(\partial_{\theta}^{k} \partial_{t} \psi\right)^{2}+\left(\partial_{\theta}^{k+1} \psi\right)^{2}\right] \mathrm{d} \theta
$$

then

$$
H_{k}\left(T_{1}\right) \leqslant \frac{\mathrm{e}}{2}\left\|\partial_{\theta}^{k} v\right\|_{L^{\infty} L^{2}\left(\mathbb{R}_{+} \times S^{1}, \mathbb{R}\right)}^{2} T_{1}^{-3} .
$$

Our algorithm for constructing the desired $\psi$ is to choose a sequence $T_{n} \rightarrow \infty$ with $T_{n} \geqslant 1$ and then define solutions $\psi^{(n)}$ to (11) by demanding that the initial data be zero at $t=T_{n}$. We then prove that $\left[\psi^{(n)}(1, \cdot), \psi_{t}^{(n)}(1, \cdot)\right]$ form a Cauchy sequence in any $C^{k}$ norm. Finally, we let $\psi$ be the solution to (11) defined by the corresponding initial data. Since the sequence $\psi^{(n)}$ satisfies bounds of form (17) in $\left[1, T_{n}\right)$, we get the conclusion that $\psi$ satisfies this bound for all future times. What remains to be done is thus to prove that the $\psi^{(n)}$ define a Cauchy sequence in the above sense.

Let $1 \leqslant T_{n} \leqslant T_{m}$ and define

$$
\tilde{\psi}=\psi^{(n)}-\psi^{(m)} \text {. }
$$

Note that $\tilde{\psi}$ satisfies (9). Let us define $\tilde{H}$ and $\tilde{\Gamma}$ as in (12) and (13) respectively, but with $\psi$ replaced with $\tilde{\psi}$. By arguments similar to those for $H$ and $\Gamma$, we get the conclusion that

$$
\frac{\mathrm{d} \tilde{\mathcal{E}}}{\mathrm{d} t} \geqslant 0
$$

where $\tilde{\mathcal{E}}$ is defined as in (16) but with $H$ and $\Gamma$ replaced with $\tilde{H}$ and $\tilde{\Gamma}$. We conclude that

$$
\tilde{H}(1) \leqslant 2 \mathrm{e} \tilde{\mathcal{E}}(1) \leqslant 2 \mathrm{e} \tilde{\mathcal{E}}\left(T_{n}\right) \leqslant 3 \mathrm{e} T_{n} \tilde{H}\left(T_{n}\right) \leqslant \frac{3 \mathrm{e}^{2}}{2}\|v\|_{L^{\infty} L^{2}\left(\mathbb{R}_{+} \times S^{1}, \mathbb{R}\right)}^{2} T_{n}^{-2},
$$

where we have used the fact that $\tilde{H}\left(T_{n}\right)$ is the energy of $\psi^{(m)}$, and the inequality (17). Note that we also have similar estimates for the derivatives. Thus the sequence $\left[\psi^{(n)}(1, \cdot), \psi_{t}^{(n)}(1, \cdot)\right]$ forms a Cauchy sequence in any $C^{k}$-norm. Let $\psi$ be the solution to (11) corresponding to the resulting smooth initial data. By the estimate (17), we get the desired decay estimates for $\psi$ and its derivatives, and by construction, $\psi$ has zero average and $P$ defined by (6) satisfies (9).

One way to obtain uniqueness is from the results of [4] or [7], however, since the proof is quite short, we shall write it down here. Assume that $P$ defined by (6) with $\psi$ replaced by $\psi_{2}$ is a solution to (9). Assume furthermore that the average of $\psi_{2}$ is zero and that its first derivatives are $o\left(t^{-1 / 2}\right)$ in $L^{2}$. We conclude that $\tilde{\psi}=\psi-\psi_{2}$ is a solution to (9) and that the first derivatives are $o\left(t^{-1 / 2}\right)$ in $L^{2}$. Just as before, we get (18). However, $\tilde{\mathcal{E}} \geqslant 0$, and due to our assumptions, $\tilde{\mathcal{E}}$ converges to zero. We conclude that $\tilde{\mathcal{E}}$ is identically zero, i.e. that $\psi=\psi_{2}$.

Finally, let us prove that (10) implies that the integral of the right-hand side of (5) is zero. Note that

$$
c_{1}=\int_{S^{1}} t\left(P_{t} P_{\theta}\right)(t, \theta) \mathrm{d} \theta, \quad \text { and } \quad c_{2}=\int_{S^{1}}\left(v_{t} v_{\theta}\right)(t, \theta) \mathrm{d} \theta
$$


are both independent of $t$ due to (2) and (7) respectively, with $Q=0$ in the former case. However, due to the fact that we have (6) where $\psi$ and its derivatives are $O\left(t^{-3 / 2}\right)$, we get the conclusion that

$$
t P_{t} P_{\theta}=v_{t} v_{\theta}+O\left(t^{-1 / 2}\right) .
$$

Consequently $c_{1}=c_{2}+O\left(t^{-1 / 2}\right)$, so that $c_{1}=c_{2}$.

Proof of corollary 1. Let us introduce the notation $\phi=t^{1 / 4} \mathrm{e}^{-\lambda / 4}$. The trace of the second fundamental form of a hypersurface of constant areal time is given by

$$
\operatorname{tr} k=-\frac{1}{4}\left(\lambda_{t}+3 t^{-1}\right) \phi .
$$

Let us specify $\alpha=\beta=0$ and $v=\sin \theta \sin t$. Then condition (10) is fulfilled, so that we get a solution to Einstein's vacuum equations corresponding to this data at infinity. Using (6), our choice of data and the fact that $\psi$ and its derivatives are $O\left(t^{-3 / 2}\right)$, we get the conclusion that

$$
\lambda_{t}=v_{t}^{2}+v_{\theta}^{2}+O\left(t^{-1}\right) \text {. }
$$

Taking into account what $v$ is, we get the conclusion that if we let $t_{l}=l \pi, \theta_{1, l}=\pi$ and $\theta_{2, l}=\pi / 2$ then

$$
\lambda_{t}\left(t_{l}, \theta_{1, l}\right)=O\left(t_{l}^{-1}\right), \quad \lambda_{t}\left(t_{l}, \theta_{2, l}\right)=1+O\left(t_{l}^{-1}\right) .
$$

If $\theta_{i} \in S^{1}, i=1,2$ and $t \in \mathbb{R}_{+}$, then

$$
\frac{\phi\left(t, \theta_{1}\right)}{\phi\left(t, \theta_{2}\right)}=\exp \left\{-\frac{1}{4}\left[\lambda\left(t, \theta_{1}\right)-\lambda\left(t, \theta_{2}\right)\right]\right\} \text {. }
$$

Note that $\lambda_{\theta}$ is bounded for $t \geqslant 1$, so that quotient (21) satisfies a uniform upper and strictly positive lower bound for $t \geqslant 1$. Combining this observation with (19) and (20), we conclude that the sequences $t_{l}$ and $\theta_{i, l}, i=1,2$ have the desired properties.

\section{References}

[1] Anderson M 2001 On long-time evolution in general relativity and geometrization of 3-manifolds Commun. Math. Phys. 222 533-67

[2] Chruściel P T et al 1990 Strong cosmic censorship in polarized Gowdy spacetimes Class. Quantum Grav. 7 1671-80

[3] Fischer A and Moncrief V 2001 The reduced Einstein equations and the conformal volume collapse of 3-manifolds Class. Quantum Grav. 18 4493-515

[4] Jurke T 2003 On the future asymptotics of polarized Gowdy $\mathbb{T}^{3}$-models Class. Quantum Grav. 20 173-91

[5] Kichenassamy S and Rendall A 1998 Analytic description of singularities in Gowdy spacetimes Class. Quantum Grav. 15 1339-55

[6] Rendall A 2000 Fuchsian analysis of singularities in Gowdy spacetimes beyond analyticity Class. Quantum Grav. 17 3305-16

[7] Ringström H 2004 On a wave map equation arising in general relativity Commun. Pure Appl. Math. 57 657-703 